\title{
Insights into fungal communities in composts revealed by 454-pyrosequencing: implications for human health and safety
}

\author{
Vidya De Gannes ${ }^{1}$, Gaius Eudoxie ${ }^{1}$ and William J. Hickey ${ }^{2 *}$ \\ 'Department of Food Production, Faculty of Food and Agriculture, University of the West Indies, St. Augustine Campus, St. Augustine, \\ Republic of Trinidad and Tobago \\ 2 O.N. Allen Laboratory for Soil Microbiology, Department of Soil Science, University of Wisconsin-Madison, Madison, WI, USA
}

\author{
Edited by: \\ Aurelio Briones, University of Idaho, \\ USA \\ Reviewed by: \\ Andrey M. Yurkov, Leibniz Institute \\ DSMZ-German Collection of \\ Microorganisms and Cell Cultures, \\ Germany \\ Claude Murat, Institut National de la \\ Recherche Agronomique, France \\ ${ }^{*}$ Correspondence: \\ William J. Hickey, O.N. Allen \\ Laboratory for Soil Microbiology, \\ Department of Soil Science, \\ University of Wisconsin-Madison, \\ 1525 Observatory Dr., Madison, \\ WI 53706, USA \\ e-mail:wjhickey@wisc.edu
}

Fungal community composition in composts of lignocellulosic wastes was assessed via 454-pyrosequencing of ITS1 libraries derived from the three major composting phases. Ascomycota represented most (93\%) of the 27,987 fungal sequences. A total of 102 genera, 120 species, and 222 operational taxonomic units (OTUs; >97\% similarity) were identified. Thirty genera predominated (ca. 94\% of the sequences), and at the species level, sequences matching Chaetomium funicola and Fusarium oxysporum were the most abundant (26 and $12 \%$, respectively). In all composts, fungal diversity in the mature phase exceeded that of the mesophilic phase, but there was no consistent pattern in diversity changes occurring in the thermophilic phase. Fifteen species of human pathogens were identified, eight of which have not been previously identified in composts. This study demonstrated that deep sequencing can elucidate fungal community diversity in composts, and that this information can have important implications for compost use and human health.

Keywords: compost, fungi, pathogens, 454-pyrosequencing, diversity

\section{INTRODUCTION}

Compost is a dynamic and complex habitat wherein microorganisms play fundamental roles. Bacterial communities in these systems have been extensively studied (Tiquia, 2005; Takaku et al., 2006; Thummes et al., 2007; Partanen et al., 2010; De Gannes et al., 2012, 2013). However, fungi are another major group of compost microbes that contribute essential decomposition processes, particularly the initial degradation of plant polymers (Sharma, 1989; Floudas et al., 2012). Relatively little is known, however, about the composition, diversity, and succession patterns of fungal communities in compost.

While compost is valued as a soil amendment, potential health hazards have also been recognized, as some saprotrophic fungi that colonize these materials are also opportunistic human pathogens. The primary risk is exposure to bio-aerosols containing compost-derived fungi, which in humans can cause mycoses of the lungs, skin, ears, sinuses, or bone marrow (Bunger et al., 2000). Most attention has focussed on Aspergillus fumigatus, the most commonly identified fungal pathogen in composts (Ryckeboer et al., 2003; Anastasi et al., 2005; Dehghani et al., 2012). But, other pathogens have also been reported, including Candida tropicalis, Candida krusei (Bonito et al., 2010), Scytalidium lignicola, and Alternaria alternata (Anastasi et al., 2005). The spectrum of saprotrophic fungi that may also be opportunistic human pathogens is much greater than the foregoing list, but their occurrence in composts has not been extensively explored.

Molecular analyses have the potential to provide new insights into the composition of fungal communities in compost.
However, to date, such analyses have indicated that fungal diversity in these systems is low. For example, Tiquia (2005) applied 18S rDNA terminal-restriction fragment length analysis for profiling of manure composts, and identified a total of 18 unique fragments, presumably indicative of 18 different phylotypes. Also, 10 genera were identified from libraries of $18 \mathrm{~S}$ rRNA genes that were cloned from a model compost of dog food-wood chips (Hansgate et al., 2005). Hultman et al. (2010) created libraries of internal transcribed spacer (ITS) region sequences from municiple waste compost, and identified 166 OTUs ( $\geq 99 \%$ similarity) from ca. 2900 clones. It's unclear if the relatively low diversity indicated by these studies accurately reflects the communities, or if it results from a limited depth of analysis. Similarly, the dynamics of fungal communities as a function of compost phase are as yet ill-defined. In principle, since most fungi are mesophiles, a reduction in their diversity might be expected as compost heat to thermophilic temperatures. But, to date, results from molecular analyses do not consistently support that concept (Tiquia, 2005; Bonito et al., 2010) and it's unclear as to the extent to which the divergent finding reflects variation in compost properties (e.g., level and duration of heating) vs. limitations of the molecular analyses applied.

Recently, knowledge of fungal communities in soils has been expanded with the advent of the use of high-throughput sequencing technologies such as 454-pyrosequencing. Compared to traditional approaches based on cloning and Sanger sequencing in terms of cost, time, and number of sequences obtained, 454pyrosequencing has proven to be a powerful alternative for the identification of a greater depth of taxa (Tedersoo et al., 2010). For 
example, Lentendu et al. (2011) demonstrated an unprecedented depth of fungal diversity in forest soil, and illustrated the strong spatial heterogeneity of fungal communities in these soils. Buee et al. (2009) were able to identify rare individuals (OTUs), which could be correlated with ecological parameters. Pyrosequencing of the nuclear ribosomal ITS region revealed fungal communities of a cork oak forest soil were distinct from those described in other soils (Orgiazzi et al., 2012). Pyrosequencing also proved useful in the examination of fungal communities related to plant disease in agriculture soils (Xu et al., 2012).

While high through-put sequencing has greatly advanced our understanding of fungal communities in soil, its application to assess these organisms in compost has not been reported. Pyrosequencing analysis could provide new insights into the composition of fungal communities in compost, and how their evolution compares to the chemical evolution of the composts. Furthermore, deep sequencing could expand the diversity of human pathogens present in compost. Thus, the goal of our study was to obtain in-depth sequence information to assess diversity and dynamics of fungal communities in three composts of lignocellulosic agricultural wastes. Fungal composition was assessed via high throughput 454-pyrosequencing of ITS1 libraries derived from each of the three major phases of the compost process. We hypothesized that community structure of each system is unique throughout the composting process, and that these systems do not converge on a common composition in the finished product. We also hypothesized that deep sequencing of these communities would reveal a greater diversity of potential pathogens than has been indicated in the literature to date.

\section{MATERIALS AND METHODS COMPOST COMPOSITION AND ANALYSES}

The compost study was described previously in De Gannes et al. (2012). In-vessel composting was done in rotary drums, in a randomized $3 \times 2$ factorial design with six treatments and two replications per treatment. Substrates used for composting were agricultural wastes ( $v i z$. rice straw, sugar cane bagasse, coffee hulls) mixed with either cow- or sheep-manure to obtain a C:N of 25-35:1.

\section{PYROSEQUENCING AND DATA ANALYSES}

Sampling and DNA extraction was described previously in De Gannes et al. (2012). Briefly, the composts were sampled at the mesophilic stage (Day 0), thermophilic stage (Day 2 for rice straw composts, Day 3 for coffee hulls composts, no sample for sugar cane bagasse) and completion of the study (mature stage, Day 82). Aliquots of the treatment replicates were pooled, and an aliquot from each of these samples was then used for PCR. For the mature phase of the bagasse and coffee composts, two replicate aliquots were used in PCR, giving a total of 10 samples analyzed by pyrosequencing. While fungal DNA recovered could have originated from spores or vegetative cells, the latter are more to be the primary source as the extraction method employed (Power Soil DNA extraction kit; MoBio), lacks the more vigorous physical disruption techniques that are often needed for rupture of fungal spores.
Fungal DNA was amplified by using primers ITS1F and ITS2 (Table S1) to generate $c a .400 \mathrm{bp}$ fragments of the nuclear ribosomal ITS1 region. Each forward primer contained the A linker for sequencing, and one of 10 unique Roche multiplex identifiers (Table S1). Thermal cycling parameters were: $94^{\circ} \mathrm{C}$ for $4 \mathrm{~min}$, $30 \mathrm{~s}$ at $94^{\circ} \mathrm{C}(30$ cycles $), 50^{\circ} \mathrm{C}$ min, $72^{\circ} \mathrm{C}$ for $90 \mathrm{~s}$, and a final extension of $10 \mathrm{~min}$ at $72^{\circ} \mathrm{C}$. Replicate PCR was done with the mature phase of the bagasse and coffee as described previously (De Gannes et al., 2012). However, technical issues occurring during sequencing resulted in no useable data being returned from one of the replicate bagasse samples. Amplicon concentrations were measured with a Qubit fluorometer (Invitrogen, Grand Island, NY), the libraries were pooled in equimolar concentrations and then cleaned by $5 \times$ passage over AMPure beads (Beckman Coulter, Brea, CA). Clonal amplified via emulsion PCR was done with the GS FLX Titanium Lib-L LV emPCR Kit (Roche Applied Science, Indiananpolis, IN) following the manufacturer's recommendations, with the exception that the amplification primer was used at $25 \%$ of the recommended volume. A DNA library was created by using a ratio of 1 DNA molecule per capture bead. Clonally amplified DNA was collected and enriched according to the manufacturer's protocol, and the number of enriched DNA beads determined with a CASY Model DT cell counter (Roche Applied Science). Beads were added to wells of a GS FLX Titanium PicoTiterPlate fitted with a 2-region gasket, and sequenced using a GS FLX Titanium Sequencing Kit $\mathrm{XL}+$ according to the manufacturer's instructions. Image analysis and signal processing were done through the shotgun/paired end signal processing pipeline in GS Run Processor v. 2.6 (Roche Applied Science). The raw data set prior to denoising was 36,314 sequences.

Data processing was done by using the ITS Pipeline (Nilsson et al., 2009). Sequence reads of $<100 \mathrm{bp}$ were deleted from the data set, and the ITS extractor used to obtain ITS1 data free of sequences mapping to the $5.8 \mathrm{~S}, 18 \mathrm{~S}$, or $28 \mathrm{~S}$ rRNA genes. The TIGR Gene Clustering Indices tool was used to create OTUs at 97\% similarity with reference to the UNITE (Abarenkov et al., 2010) and INSD (Karsch-Mizrachi et al., 2011) databases. Species level identities were confirmed by manual BLAST against the Genbank non-redundant data base followed by inspection to verify a full length match of $\geq 97 \%$ to a sequence of a bona fide fungal culture. Denoising of the dataset, rarefaction analysis by a radomization procedure that included singletons was done with QIIME v. 1.5.0 (Caporaso et al., 2010). Rank abundance was calculated in Excel, and plotted with Prism v. 5.0 (GraphPad Software, Inc., La Jolla, CA). The relative abundance of a given taxon was equated with relative abundance of sequence reads, assuming the caveats delineated by Amend et al. (2010). Furthermore, while the ITS region is widely used for species delimitation, it is also recognized that ITS sequences are insufficient to resolve some fungal species and species complexes, and species assignments are made recognizing that limitation. Also, either ITS1 or ITS2 could be used for analysis of fungal communities, each has benefits and drawbacks and the selection largely dependent upon the goals of the study (Bazzicalupo et al., 2013). For the present work, ITS1 was selected, as there is a somewhat larger use of that region in the literature, and to which the present work would be comparable (Chen et al., 
2001; Narutaki et al., 2002; Hinrikson et al., 2005; Nilsson et al., 2008).

\section{SEQUENCE ACCESSION NUMBERS}

Sequence data have been deposited in National Center for Biotechnology Information, Sequence Read Archive (SRX079878).

\section{RESULTS}

\section{CHEMICAL PROPERTIES OF COMPOSTS}

Chemical properties of the composts were discussed previously in De Gannes et al. (2012). Briefly, by day 2, the rice composts attained a thermophilic temperature of $\mathrm{ca} .57^{\circ} \mathrm{C}$, and remained at that temperature for 3-4 days before the onset of cooling. The coffee composts attained a peak thermophilic temperature of $64^{\circ} \mathrm{C}$ by day eight, but maintained thermophilic temperature for ca. 5 days. The sugar cane bagasse compost reached a peak temperature of $c a .38^{\circ} \mathrm{C}$, which was not considered a thermophilic stage (minimum $50^{\circ} \mathrm{C}$ ). All composts showed reductions in total organic carbon (TOC) with time. However, rice showed the fastest rate of decrease. At the end of the study (day 82 ), TOC for rice, bagasse, and coffee were ca. $22 \mathrm{mg} / \mathrm{kg}$, ca. $26 \mathrm{mg} / \mathrm{kg}$, and $\mathrm{ca} .28 \mathrm{mg} / \mathrm{kg}$, respectively. The rice compost had a final C:N of 11, while that of the coffee and bagasse was $c a$. 15 and 17, respectively. The dynamics of nitrogen transformations also varied in these composts. For the bagasse and rice, nitrate increased steadily during the course of the study and by its end, the nitrate content for bagasse was $c a .100 \mathrm{mg} / \mathrm{kg}$ and that for rice was ca. $120 \mathrm{mg} / \mathrm{kg}$. Nitrate accumulation for the coffee, however, was slower reaching $30 \mathrm{mg} / \mathrm{kg}$ at the end of the study. Each compost also showed a unique $\mathrm{pH}$ profile. The $\mathrm{pH}$ of coffee was initially at 5-6 but increased to $\mathrm{ca}$. 7.4 by the end of the study. Bagasse also had an initial $\mathrm{pH}$ of 56, which spiked to $\mathrm{pH} 7-8$ at the third week and then returned to $\mathrm{pH} 5-6$ at the end (day 82). Contrastingly, the rice was initially alkaline ( $\mathrm{pH} 8)$, but by the end of the study decreased to a $\mathrm{pH}$ of 6-7.

\section{LIBRARY COMPOSITION AND FUNGAL DIVERSITY}

A total of 28,145 sequences were returned via ITS Pipeline processing and, after removal of 158 sequences matching animal taxa, the fungal sequences totaled 27,987 (Table 1, Table S2). While it's possible that the primers could also amplify the ITS from plants, no plant sequences were identified. A total of 222 OTUs were identified with the number in any given library ranging from 39 to 149 (Table 1). Only six OTUs were identified in all libraries (Table S2), which matched to Chaetomium funicola (OTU 350),
Aspergillus oryzae (OTU 38), Thermomyces lanuginosus (OTU 123, OTU 59), and Fusarium oxysporum (OTU 506, OTU 83). Rarefaction curves for most libraries approached a plateau or were asymptotic (Figure 1). Those for mesophilic bagasse and mature phase rice were truncated because of the limited number of sequences obtained, nevertheless, these samples paralleled curves of the other more extensively sequenced libraries, suggesting a similar trend. Overall, rarefaction analyses indicated that the libraries provided an adequate sampling of fungal diversity in the composts.

Rank-abundance plots displayed contrasting patterns of species richness and at the same time, highlighted evenness among assemblages (Tokeshi, 1993; Smith and Wilson, 1996) (Figures 2A-C). In the coffee compost, the community of the thermophilic phase showed the lowest species evenness (lowest curve) and also the lowest species richness (shortest curve). By the mature phase, both the mesophilic and thermophilic parameters increased (Figure 2A). All phases were dominated by one or two OTU, as illustrated in a sharp drop in abundance from the top-ranks (Figure 2A). But, the gap between the dominant OTU and the next tier of OTUs narrowed in the mature phase, indicating proliferation of sub-dominant groups. In contrast, for the rice compost, species evenness increased after the mesophilic phase to similar levels in the thermophilic and mature stages (Figure 2B). Species richness, however, reached a maximum in

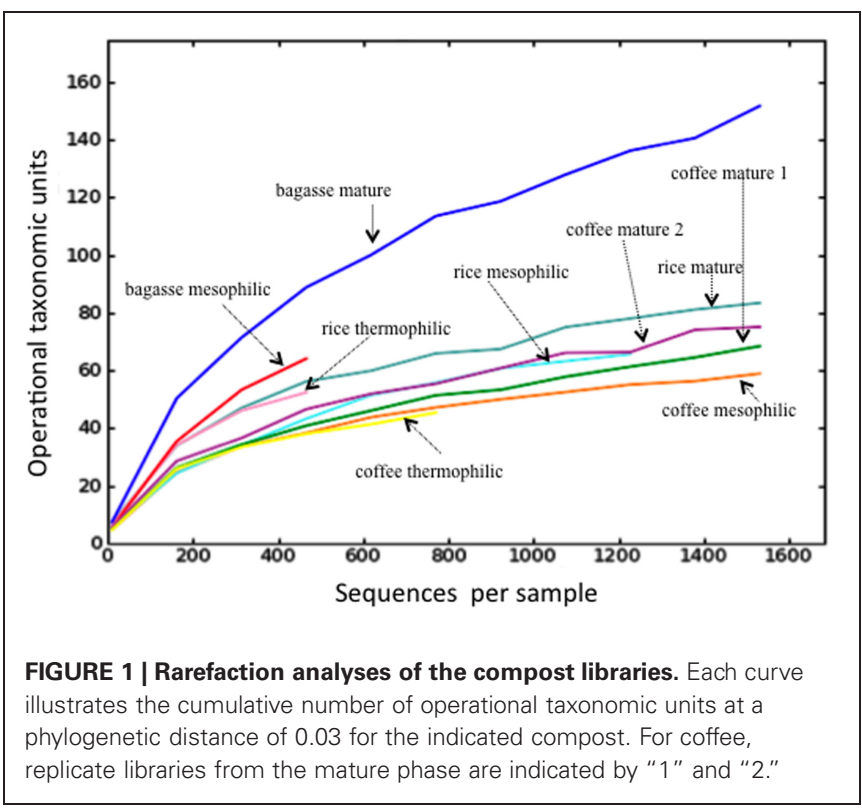

Table 1 | Characteristics of amplicon libraries ${ }^{a}$.

\begin{tabular}{llllllllll}
\hline & BM & BMT & CM & CT & CMT.1 & CMT.2 & RM & RT & RMT \\
\hline No. of sequences & 522 & 6557 & 1509 & 7225 & 5983 & 892 & 1340 & 580 & 3537 \\
No. of OTU & 44 & 148 & 39 & 88 & 88 & 92 & 50 & 49 & 78 \\
\hline
\end{tabular}

aAbbreviations: B, Bagasse; C, Coffee; R, Rice; M, Mesophilic; T, Thermophilic; MT, Mature.

${ }^{b}$ Total number of OTU determined for the entire database (see Table S2). 


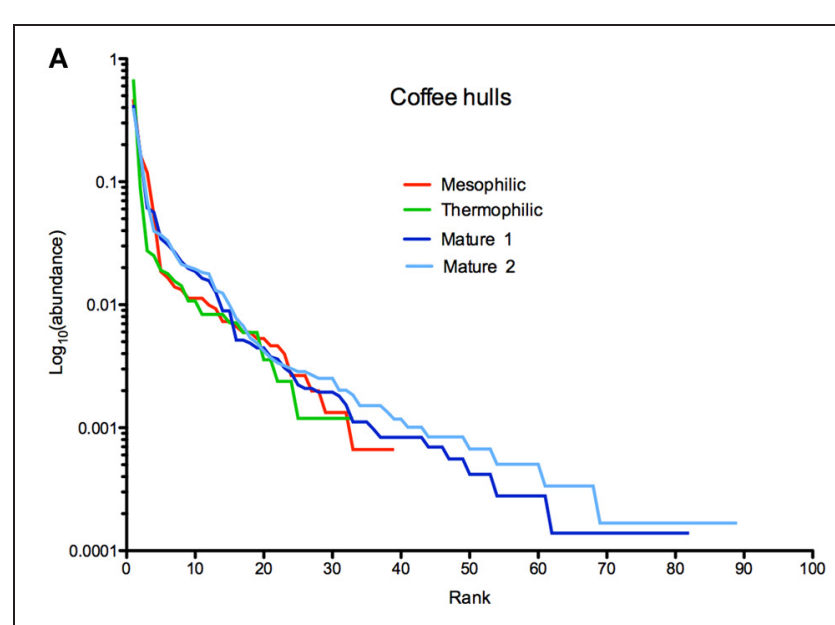

B

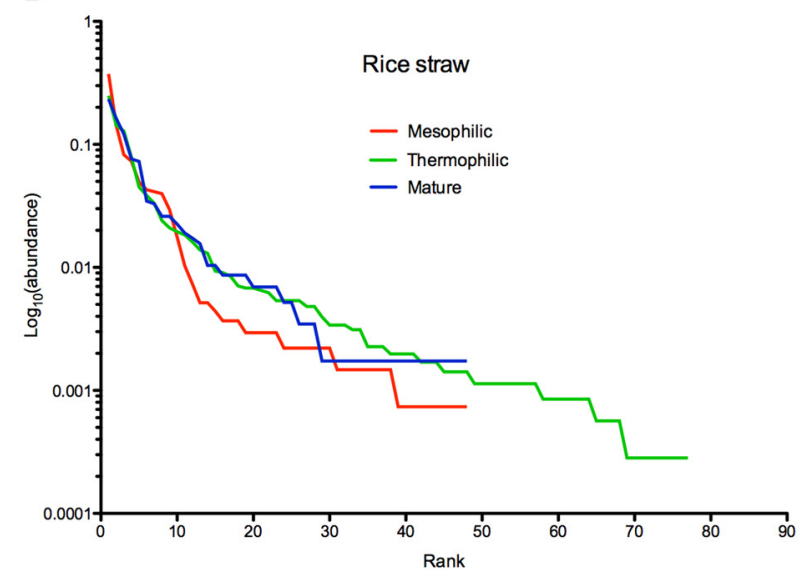

C

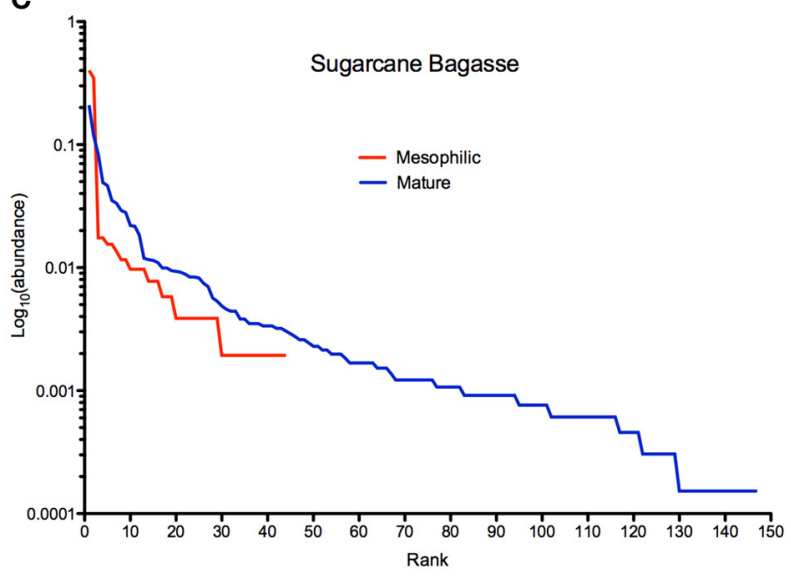

FIGURE 2 | Rank abundance plots of fungal community composition in the coffee (Panel A), rice (Panel B), and bagasse (Panel C) composts.

the thermophilic phase, and in the mature phase decreased to levels similar to those occurring at the beginning of the study. The bagasse system showed the greatest alterations in community composition, and by the end of the study attained the highest level of species richness observed in any of the composts.

\section{TAXONOMIC COMPOSITION OF COMMUNITIES}

A total of 102 genera and 120 species were identified (Table S2) with 30 genera accounting for ca. 94\% of the sequences (Table 2, Figure 3). In some instances, more than one OTU corresponded to the same species (Table S2). Most of these species were from phylum Ascomycota and class Sordariomycetes, which was the predominant phylum and class in all compost types and phases (93\% of the sequences, Table S2). The Basidiomycota and Zygomycota accounted for 6 and $1 \%$ of the total sequences, respectively. At the species level, sequences that matched to Chaetomium funicola and Fusarium oxysporum were the most abundant, accounting for 26 and 12\%, respectively of all sequence reads (Table S2). It should be noted that only a few species of fungi are known thermophiles and can survive the thermophilic temperature stage. In this regard only few fungi identified in this study are known thermophiles and they include Aspergillus fumigatus, Scytalidium thermophilum, and Thermomyces lanuginosus. Thus, this reason (i.e., small number of thermophilic fungi detected) and also the dynamic shifts in fungal diversity from the beginning to the end of the composting study for each system (even though it is difficult to discern whether or not the fungal communities detected were living organisms or resting spores), suggests that there was turn over in the DNA. In this regard, if they were merely resting spores, then those fungi which were detected alone in the beginning of the study would have been detected alone throughout the experiment.

Each compost had a unique community composition and community dynamic. In the coffee compost, Fusarium, Aspergillus, and Eurotium were the predominant genera in the mesophilic phase library and accounted for 59, 18, and $6 \%$ of the sequences, respectively (Figure 3). In the thermophilic stage, Fusarium and Aspergillus remained the most abundant and accounted for 69 and $4 \%$, of the sequences, respectively (Figure 3). However, Eurotium was displaced by Monascus (9\%, Figure 3). In the mature stage, a new set of genera predominated, which included Chaetomium, Monacrosporium, Scytalidium, and Hyaloscypha (Figure 3). In the rice compost, three genera, Scytalidium, Chaetomium, and Fusarium, were predominant throughout. Of these, Scytalidium was consistently most abundant, and accounted for 52, 21, and $22 \%$ of the sequences in the mesophilic-, thermophilic-, and mature-stages, respectively (Figure 3). In the mesophillic bagasse compost, Scedosporium and Fusarium accounted for most of the sequences (42 and $38 \%$, respectively). But, by the end of the process, these genera were replaced by Scytalidium, Chaetomium, and Gleophyllum (Figure 3). It should be noted that, as with any environmental analysis, spatial variability in the composts could affect the results such that the community composition of any given sample could differ to some extent from any other sample. Thus, the aforegoing discussion of the communities determined as representative of the compost types and phases is presented with this caveat.

\section{POTENTIAL PATHOGENS IN COMPOSTS}

Fifteen known fungal human pathogenic species were identified, and included seven that have been reported by prior investigators in composts (Alternaria alternata, Aspergillus fumigatus, Candida tropicalis, Chaetomium funicola, Cladosporium cladosporioides, 
Table 2 | Most abundant fungal genera identified for the predominant genera composing compost libraries ${ }^{\mathrm{a}}$.

\begin{tabular}{|c|c|c|c|c|c|}
\hline Phylum & Class & Order & Family & Genus & $\%$ Total $^{b}$ \\
\hline Ascomycota & Sordariomycetes & Sordariales & Chaetomiaceae & Chaetomium & 28.5 \\
\hline Ascomycota & Leotiomycetes & Helotiales & Incertae sedis & Scytalidium & 16.3 \\
\hline Ascomycota & Sordariomycetes & Hypocreales & Nectriaceae & Fusarium & 12.1 \\
\hline Ascomycota & Orbiliomycetes & Orbiliales & Orbiliaceae & Monacrosporium & 8.7 \\
\hline Ascomycota & Sordariomycetes & Microascales & Microascaceae & Scedosporium & 3.8 \\
\hline Ascomycota & Leotiomycetes & Helotiales & Hyaloscyphaceae & Hyaloscypha & 3.3 \\
\hline Basidiomycota & Agaricomycetes & Gleophyllales & Cloeophyllaceae & Gleophyllum & 3 \\
\hline Ascomycota & Sordariomycetes & Sordariales & Chaetomiaceae & Corynascus & 3 \\
\hline Ascomycota & Sordariomycetes & Microascales & Microascaceae & Graphium & 2.4 \\
\hline Ascomycota & Eurotiomycetes & Eurotiomycetes & Eurotiales & Aspergillus & 2.2 \\
\hline Ascomycota & Eurotiomycetes & Eurotiales & Monascaceae & Monascus & 1.8 \\
\hline Ascomycota & Incerate sedis & Incerate sedis & Incerate sedis & Retroconis & 1.2 \\
\hline Zygomycota & Incerate sedis & Mottierellales & Mortierellaceae & Mortierella & 1 \\
\hline Basidiomycota & Agaricomycetes & Agaricales & Agaricaceae & Coprinus & 0.9 \\
\hline Ascomycota & Sordariomycetes & Hypocreales & Clavicipitaceae & Metarhizium & 0.8 \\
\hline Ascomycota & Sordariomycetes & Sordariales & Chaetomiaceae & Humicola & 0.8 \\
\hline Ascomycota & Sordariomycetes & Chaetothyriales & Herpotrichiellaceae & Cladophialophora & 0.7 \\
\hline Ascomycota & Eurotiomycetes & Eurotiales & Trichocomaceae & Penicillium & 0.6 \\
\hline Ascomycota & Eurotiomycetes & Sordariales & Cephalothecaceae & Cephalotheca & 0.6 \\
\hline Ascomycota & Sordariomycetes & Orbiliales & Orbiliaceae & Arthrobotrys & 0.5 \\
\hline Ascomycota & Orbiliomycetes & Peltigerales & Peltigeraceae & Peltigera & 0.4 \\
\hline Ascomycota & Lecanoromycetes & Eurotiales & Trichocomaceae & Eurotium & 0.4 \\
\hline Basidiomycota & Urediniomycetes & Sporidiobolales & Sporidiobolaceae & Rhodotorula & 0.1 \\
\hline Ascomycota & Sordariomycetes & Xylariales & Xylariaceae & Thielavia & 0.1 \\
\hline Ascomycota & Sordariomycetes & Microascales & Microascaceae & Verticillium & 0.09 \\
\hline Basidiomycota & Sordariomycetes & Russulales & Stereaceae & Acanthophysium & 0.07 \\
\hline Basidiomycota & Agaricomycetes & Polyporales & Polyporaceae & Trametes & 0.06 \\
\hline Basidiomycota & Agaricomycetes & Agaricales & Schizophyllaceae & Schizophyllum & 0.06 \\
\hline Basidiomycota & Agaricomycetes & Polyporales & Polyporaceae & Polyporus & 0.05 \\
\hline Basidiomycota & Agaricomycetes & Agaricales & Schizophyllaceae & Chrondrostereum & 0.03 \\
\hline
\end{tabular}

a Thirty predominant fungal genera shown in Figure 3.

${ }^{b}$ Percent of sequence reads in all libraries (27,987 total).

Fusarium oxysporum, Scytalidium lignicola) as well as eight that have not been previously reported in such systems (Bipolaris spicifera, Fonsecaea pedrosoi, Metarhizium anisopliae, Retroconis fusiformis, Scedosporium apiospermum, Scedosporium aurantiacum, Scedosporium prolificans, and Cladophialophora arxii). Seven of these species (Alternaria alternata, Aspergillus fumigatus, Chaetomium funicola, Cladosporium cladosporioides, Fusarium oxysporum, Scedosporium aurantiacum, and Cladophialophora arxii) were identified in all composts, five species (Metarhizium anisopliae, Retroconis fusiformis, Scedosporium apiospermum, Scytalidium lignicola, and Scedosporium prolificans) were present in both bagasse and coffee composts, and three were detected in bagasse alone (Bipolaris spicifera, Fonsecaea pedrosoi, and Candida tropicalis).

\section{DISCUSSION}

The rank-abundance data showed that, in the coffee system, diversity decreased between the mesophilic and thermophilic phases, and then increased to its highest levels in mature phase.
The rice compost showed a contrasting pattern with diversity increasing to its maximum level in the thermophilic phase, and then declining in the mature phase. The bagasse system, which lacked a thermophilic phase, showed the greatest expansion in fungal diversity from the mesophilic to mature stage, and thus achieved the highest level of diversity overall. These findings supported the concept of fungal community successions occurring across compost phases (Ryckeboer et al., 2003). But, the data did not consistently support prior findings that the occurrence or extent of a thermophilic phase to be the most important factor affecting the levels of diversity in the finished product (mature phase).

Previous studies have shown that fungal diversity decreased at thermophilic temperatures (Ryckeboer et al., 2003; Tiquia, 2005; Bonito et al., 2010; Hultman et al., 2010). But for the composts which attained thermophilic temperatures in the present study (rice and coffee), only the coffee was consistent with this finding. A possible explanation for the divergent behavior of fungal communities in these composts could have been the level of heat 


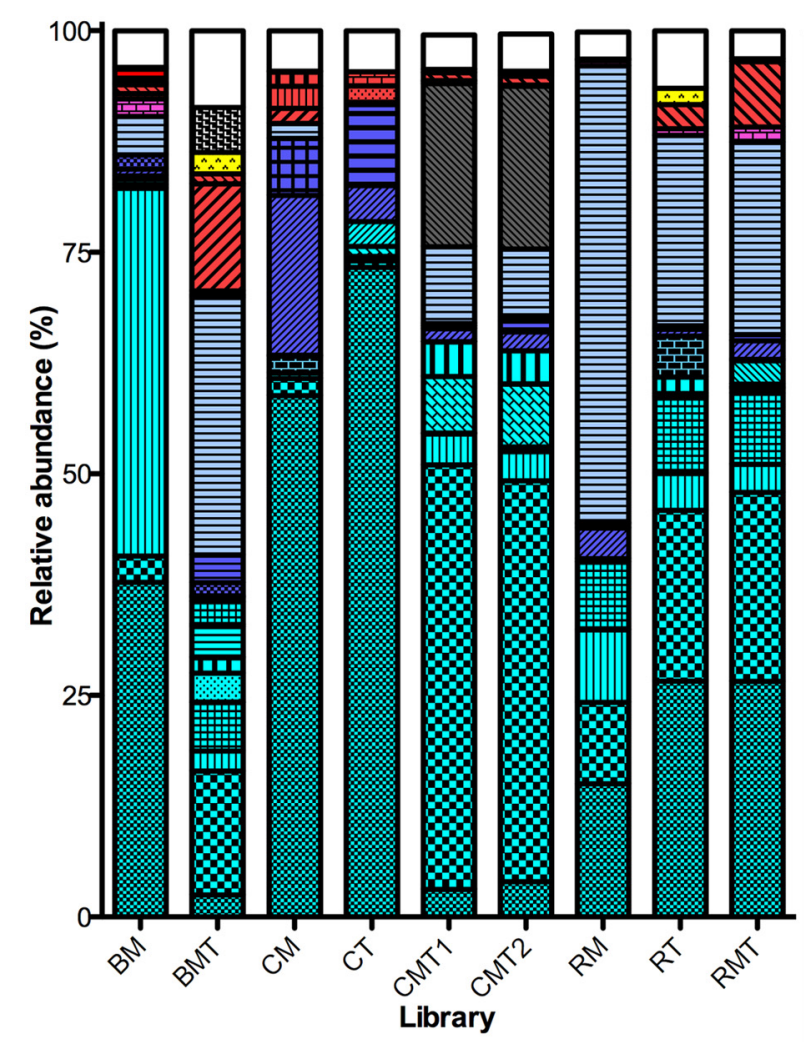

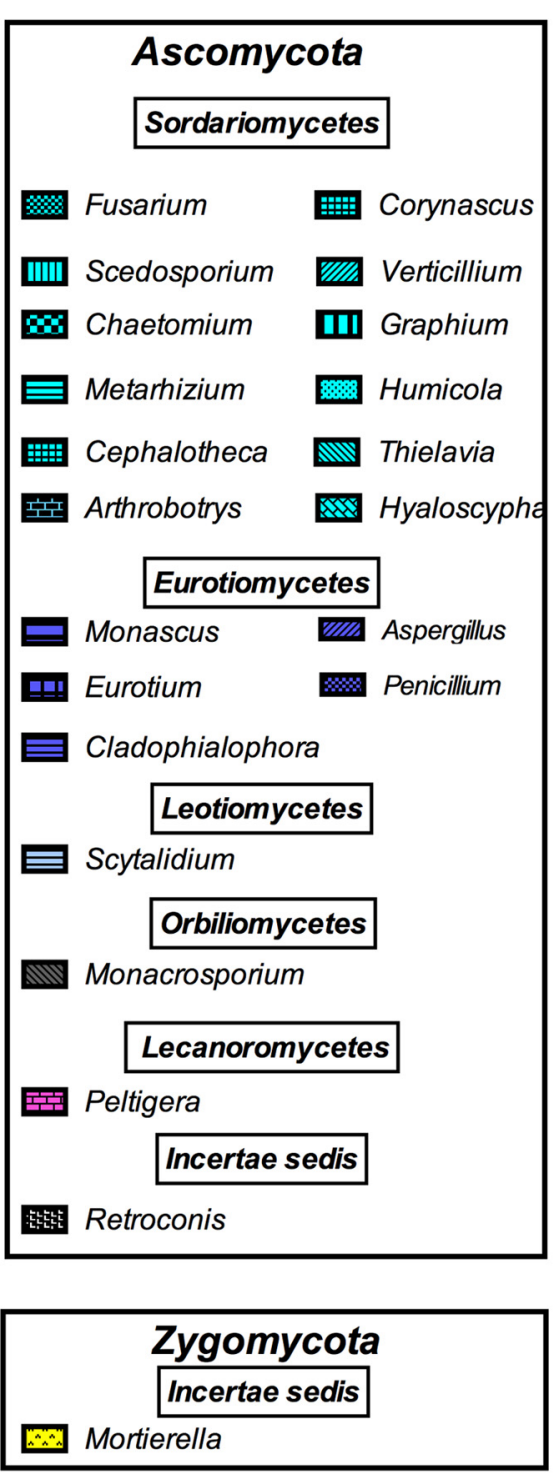

FIGURE 3 | Composition of compost sequence libraries illustrating the distribution of $\mathbf{3 0}$ genera that accounted for $94 \%$ of the sequences in the entire dataset. The segments composing each bar are the number of sequences matching the indicated genus normalized to the total number of sequences in each library. Genera are sorted by phylum (boxes in figure), bars are color-coded by class as indicated.
White boxes are the sum of all other genera in each library. Abbreviations are as follows: BM, bagasse mesophilic phase; BMT, bagasse mature phase; $\mathrm{CM}$, coffee mesophilic phase; $\mathrm{CT}$, coffee thermophilic phase; CMT1, coffee mature phase replicate 1; CMT2, coffee mature phase replicate 2; RM, rice mesophilic phase; $\mathrm{RT}$, rice thermophilic phase; $\mathrm{RMT}$, rice mature phase. generated and duration of the thermophilic phase. In this regard, the coffee compost reached and maintained $64^{\circ} \mathrm{C}$ for $5 \mathrm{~d}$, while the rice system heated to $57^{\circ} \mathrm{C}$ for 3-4 d. Notably the bagasse system, which never attained a thermophilic temperature exhibited the greatest expansion in fungal diversity. This could reflect the fact that most terrestrial fungi are mesophiles (Dix and Webster, 1995), and maintenance of mesophilic conditions in the bagasse could have allowed their undisrupted growth, and consequently development of communities more diverse than those of the other composts. Previously we reported analysis of prokaryotic diversity in the same bagasse, coffee, and rice composts (De Gannes et al., 2013), and comparison of that data with the present study shows that prokaryotic diversity was far greater than that of fungi. This finding was congruent with those of other investigators (Ryckeboer et al., 2003; Tiquia, 2005).

The prevalence of Fusarium might be anticipated given its ubiquity both in soil and association with plants. But, the relative abundance of Fusarium may to some extent have been an indicator of compost maturity, as its sequences tended to be greatest in meso- and thermo-philic phases (Table S2). Thus, mature phase compost was characterized by a reduction in Fusarium. All Fusarium sequences were matched to Fusarium 
oxysporum (Table S2), strains of which exhibit a variety of activities that range from lignin degradation (Kadarmoidheen et al., 2012) to causing plant (Beckman, 2000), animal (Odds et al., 1998), and human diseases (Boutati and Anaissie, 1997). Scytalidium was also prevalent in the composts, and the two species identified, Scytalidium thermophilum and Scytalidium lignicola, were divergently distributed (Table S2). The former of these was predominantly associated with the coffee and rice composts, and in the coffee system showed a striking increase in abundance in the mature phase (Table S2). Prior investigators have noted that this thermophilic fungus often exhibits profuse growth in mature phase compost from spores produced during thermophilic phase growth (Wiegant, 1992). In contrast, the majority of Scytalidium lignicola sequences were identified in the mature phase of the bagasse compost (Table S2), which lacked a thermophilic phase. The two species of Chaetomium identified, Chaetomium funicola and Chaetomium globosum, showed a similar bipartite distribution with the thermophilic organism, Chaetomium funicola, occurring primarily in rice and coffee composts (especially the coffee mature phase) while abundance of the mesophilic Chaetomium globosum was heavily weighted toward the mature phase bagasse (Table S2).

Prior studies have provided insights about the presence of opportunistic pathogens associated with composting systems, viz. Candida tropicalis and Candida krusei (Bonito et al., 2010), Aspergillus fumigatus (Dehghani et al., 2012), Scytalidium lignicola, and Alternaria alternata (Anastasi et al., 2005). However, the deep sequencing presented here has revealed the broadest spectrum of potentially pathogenic fungal species yet reported for a composting study. The group of eight species detected in all composts includes all but one of the potential pathogens identified by prior investigators, and thus could be representative of the main types of potential pathogens that might be encountered in composts. If so, bio-monitoring efforts could be expanded beyond Aspergillus fumigatus to include these organisms as well.

Fungal pathogens are an increasing threat to human health, and those listed above are known to cause several types of mycoses, with immune-compromised individuals being particularly susceptible. Allergic broncho-pulmonary aspergillosis, caused primarily by Aspergillus fumigatus (Latge, 1999; Chakrabarti et al., 2002; Hope et al., 2005; Agarwal et al., 2009) is a wide-spread concern (Howard et al., 2010; Denning et al., 2011). Skin diseases are also common and include phaeohyphomycosis caused by Scytalidium lignicola, Candida tropicalis (Wingard et al., 1979), Scedosporium prolificans (Revankar et al., 2002), and Bipolaris spicifera (McGinnis et al., 1992) as well as chromoblastomycosis, which have involved Fonsecaea pedrosoi and Chaetomium funicola (Pipenbring et al., 2007). The latter affliction is common in tropical and subtropical regions (Silva et al., 2002).

The clinical spectrum for most of these fungal pathogens is diverse. For example, Bipolaris spicifera is a source of nosocomial infections and has also caused a type of meningitis (Ogden et al., 1992) as well as cutaneous and pulmonary infections (McGinnis et al., 1992). Scedosporium prolificans have been shown to cause ocular infections (keratouveitis; Arthur et al., 2001) as well as cases of pneumonia (Berenguer et al., 1997); treatment of Scedosporium prolificans infections are difficult because of its resistance to many commonly used antifungal agents. Scedosporium apiospermum causes a range of infections such as bullous and necrotic purpura (Miyamoto et al., 1998), osteomyelitis (German et al., 2004) while infection by Scedosporium aurantiacum can result in malignant otitis externa, osteomyelitis, invasive sinusitis, keratitis, and pneumonia (Alastruey-Izquierdo et al., 2007). Concerns with Fusarium oxysporium include production of a range of mycotoxins (trichothecenes, zearalenon, and fumonisins) in addition to infections ranging from skin lesions to allergies (Boutati and Anaissie, 1997). Alternaria alternata also produces mycotoxins that caused allergies and asthma in children (Halonen et al., 1997). Infections caused by Cladophialophora arxii are chronic and appear as spreading mycoses of the skin, subcutaneous tissues, and nail (Barde and Singh, 1984).

According to the American Biological Safety Association, fourteen of the fungal pathogens identified in this study are categorized as Biosafety Level 2 (Alternaria alternate, Aspergillus fumigatus, Candida tropicalis, Chaetomium funicola, Cladosporium cladosporioides, Fusarium oxysporum, Scytalidium lignicola, Bipolaris spicifera, Fonsecaea pedrosoi, Metarhizium anisopliae, Retroconis fusiformis, Scedosporium apiospermum, Scedosporium aurantiacum, Scedosporium prolificans) and one (Cladophialophora arxii) belonged to Biosafety Level 3. Hence, it is recommended that personal protective equipment be worn when handling these composts. These include face protection (goggles, face masks), disposable gloves, and protective coats. It should be noted that, for all of the foregoing species, there can exist substantial intra-specific (strain level) variation in virulence (Ben-Ami et al., 2010). Thus, molecular detection such as that of the present study can provide an indicator of the potential health threat, but additional bioassays of isolates are needed to assess virulence.

\section{CONCLUSIONS}

Results of the study supported both hypotheses. First, the community structure of each system was unique throughout the composting process, and did not converge on a common composition in the finished product. Thus, there was no single fungal community structure that could be considered typical. Second, the diversity of potential pathogens was greater than previously reported. Molecular identification of these indicated that safety precautions would be warranted for both compost producers and users. Overall, the study demonstrated that deep sequencing can effectively elucidate fungal community diversity in compost and that such information can have important implications for compost use and human health.

\section{ACKNOWLEDGMENTS}

These studies were supported by a grant (to Vidya De Gannes) from the Postgraduate Research Fund and the Department of Food Production, Faculty of Food and Agriculture University of the West Indies, St. Augustine Campus, Trinidad and Tobago, and an endowment from the O.N. Allen Professorship in Soil 
Microbiology (to William J. Hickey). The authors are grateful to the academic and technical staff from the Department of Food Production, Faculty of Food and Agriculture and Department of Life Sciences, Faculty of Science and Technology, The University of the West Indies, St. Augustine Campus, Trinidad and Tobago.

\section{REFERENCES}

Abarenkov, K., Nilsson, R. H., Larsson, K. H., Alexander, J. I., Eberhardt, U., Erland, S., et al. (2010). The UNITE database for molecular identification of fungi-recent updates and future perspectives. New Phytol. 186, 281-285. doi: 10.1111/j.14698137.2009.03160.x

Agarwal, R., Aggarwal, A. N., Gupta, D., and Jindal, S. K. (2009). Aspergillus hypersensitivity and allergic bronchopulmonary aspergillosis in patients with bronchial asthma: systematic review and meta-analysis. Int. J. Tuberc. Lung Dis. 13, 936-944.

Alastruey-Izquierdo, A., CuencaEstrella, M., Monzón, A., and Rodriguez-Tudela, J. L. (2007). Prevalence and susceptibility testing of new species of Pseudallescheria and Scedosporium in a collection of clinical mold isolates. Antimicrob. Agent. Chemother. 51, 748-751. doi: 10.1128/AAC.01177-06

Amend, A. S., Seifert, K. A., and Bruns, T. D. (2010). Quantifying microbial communities with 454 pyrosequencing: does read abundance count? Mol. Ecol. 19, 5555-5565. doi: 10.1111/j.1365-294X.2010.04898.x

Anastasi, A., Varese, G. C., and Marchisio, V. F. (2005). Isolation and identification of fungal communities in compost and vermicompost. Mycologia 97, 33-44. doi: 10.3852/mycologia.97.1.33

Arthur, S., Steed, L. L., Apple, J. D., Peng, Q., Howard, G., and Escobar-Gomez, M. (2001). Scedosporium prolificans Keratouveitis in association with a contact lens retained intraocularly over a long term. J. Clin. Microbiol. 39, 4579-4582. doi: 10.1128/JCM.39.12.4579-4582.2001

Barde, A. K., and Singh, S. M. (1984). Cladosporium carrionii Trejos 1954 infection of human nail. Mykosen 27, 366-369. doi: 10.1111/j.14390507.1984.tb02044.x

Bazzicalupo, L. A., Balint, M., and Schmitt, I. (2013). Comparison of ITS1 and ITS2 rDNA in 454 sequencing of hyperdiverse fungal communities. Fungal Ecol. 6, 102-109. doi: 10.1016/j.funeco.2012.09.003

Beckman, H. C. (2000). Phenolicstoring cells: keys to programmed cell death and periderm formation in wilt disease resistance and in general defence responses in plants? Physiol. Mol. Plant Pathol. 57, 101-110. doi: 10.1006/pmpp.2000.0287

Ben-Ami, R., Lamaris, G. A., Lewis, R. E., and Kontoyiannis, D. P. (2010). Interstrain variability in the virulence of Aspergillus fumigatus and Aspergillus terreus in a TOU-deficient Drosophilia fly model of invasive aspergillosis. Med. Mycol. 48, 310-317. doi: 10.3109/13693780903 148346

Berenguer, J., Rodríguez-Tudela, L. J., Richard, C., Alvarez, M., Sanz, M. A., Gaztelurrutia, L., et al. (1997). Deep infections caused by Scedosporium prolificans: a report on 16 cases in Spain and a review of the literature. Medicine 76, 256-265. doi: 10.1097/00005792199707000-00004

Bonito, G., Isikhuenmhen, O. S., and Vilgalys, R. (2010). Identification of fungi associated with municipal compost using DNA-based techniques. Bioresour. Technol. 101, 1021-1027. doi: 10.1016/j.biortech.2009.08.109

Boutati, I. E., and Anaissie, J. E. (1997). Fusarium, a significant emerging pathogen in patients with hematologic malignancy: ten years' experience at a cancer center and implications for management. Blood 90, 999-1008.

Buee, M., Reich, M., Murat, C., Morin, E., Nilsson, R. H., Uroz, S., et al. (2009). 454 pyrosequencing analysis of forest soils reveal an unexpectedly high fungal diversity. New Phytol. 184, 449-456. doi: 10.1111/j.14698137.2009.03003.x

Bunger, J., Antlauf-Lammers, M., Schuliz, T., Westphal, G., Muller, M., Ruhnau, P., et al. (2000). Health complaints and immunological markers of exposure to bioaerosols among biowaste collectors and compost workers. Occup. Environ. Med. 57, 458-464. doi: 10.1136/oem. 57.7.458

Caporaso, J. G., Kuczynski, J., Stombaugh, J., Bittinger, K., Bushman, F. D., Costello, E. K., et al. (2010). QIIME allows analysis of high throughput community

\section{SUPPLEMENTARY MATERIAL}

The Supplementary Material for this article can be found online at: http://www.frontiersin.org/Microbiotechnology, Ecotoxicology_and_Bioremediation/10.3389/fmicb.2013.00164/ abstract

sequencing data. Nat. Methods 7 , 335-336. doi: 10.1038/nmeth.f.303

Chakrabarti, A., Sethi, S., Raman, D. S. V., and Behera, D. (2002). Eight-year study of allergic bronchopulmonary aspergillosis in an indian teaching hospital. Mycoses 45, 295-299. doi: 10.1046/j.14390507.2002.00738.x

Chen, Y.-C., Eisner, D. J., Kattar, M. M. Rassoulian-Barrett, L. S., Lafe, K., Bui, U., et al. (2001). Polymorphic internal transcribed spacer region 1 DNA sequences identify medically important yeasts. J. Clin. Microbiol. 39, 4042-4051. doi: 10.1128/JCM.39.11.4042-4051.2001

De Gannes, V., Eudoxie, G., Dyer, D. H., and Hickey, W. J. (2012). Diversity and abundance of ammonia oxidizing archaea in tropical compost systems. Front. Microbiol. 3:244. doi 10.3389/fmicb.2012.00244

De Gannes, V., Eudoxie., G. and Hickey, W. J. (2013) Prokaryotic successions and diversity in composts as revealed by 454-pyrosequencing. Bioresour. Technol. 133, 573-580. doi: 10.1016/j.biortech.2013.01.138

Dehghani, R., Asadi, M. A., Charkhloo, E., Mostafaie, G., Saffari, M. Mousavi, G. A., et al. (2012). Identification of fungal communities in producing compost by windrow method. J. Environ. Prot. 3 , 61-67. doi: 10.4236/jep.2012.31008

Denning, W. D., Park, S., Lass-Florl, C., Fraczek, G. M., Kirwan, M., Gore, R., et al. (2011). High-frequency triazole resistance found in nonculturable Aspergillus fumigatus from lungs of patients with chronic fungal disease. Clin. Infect. Dis. 52, 1123-1129. doi: $10.1093 / \mathrm{cid} /$ cir179

Dix, N. J., and Webster, J. (1995). Fungal Ecology. Cambridge: Chapman and Hall. doi: 10.1007/978-94-011-0693-1

Floudas, D., Binder, M., Riley, R., Barry, K., Blanchette, A. R., Henrissat, B. et al. (2012). The Paleozoic origin of enzymatic lignin decomposition reconstructed from 31 fungal genomes. Science 336, 1715. doi: 10.1126/science. 1221748

German, W. J., Kellie, M. S., Pai, P. M., and Turner, T. P. (2004). Treatment of a chronic Scedosporium apiospermum vertebral osteomyelitis.
Neurosurg. Focus 17:E9. doi: 10.3171/foc.2004.17.6.9

Halonen, M., Stern, D. A., Wright, A. L., Taussig, L. M., and Martinez, F. D. (1997). Alternaria as a major allergen for asthma in children raised in a desert environment. Am. J. Respir. Crit. Care Med. 155, 1356-1361. doi: 10.1164/ajrccm.155.4.9105079

Hansgate, A. M., Schloss, P. D. Anthony, G. H., and Walker, L. P. (2005). Molecular characterization of fungal community dynamics in the initial stages of composting. FEMS Microbiol. Ecol. 51, 209-214. doi: 10.1016/j.femsec.2004.08.009

Hinrikson, P. H., Hurst, F. S., Lott, J. T., Warnock, W. D., and Morrison, J. C. (2005). Assessment of ribosomal large-subunit D1-D2, internal transcribed spacer 1, and internal transcribed spacer 2 regions as targets for molecular identification of medically important Aspergillus species. J. Clin. Microbiol. 43, 2092-2103. doi: 10.1128/JCM.43.5.2092-2103.2005

Hope, W. W., Walsh, T. J., and Denning, D. W. (2005). Laboratory diagnosis of invasive aspergillosis. Lancet Infect. Dis. 5, 609-622. doi: 10.1016/S1473-3099(05)70238-3

Howard, S. J., Pasqualotto, A. C., and Denning, D. W. (2010). Azole resistance in allergic bronchopulmonary aspergillosis and Aspergillus bronchitis. Clin. Microbiol. Infect. 16, 683-688. doi: 10.1111/j.14690691.2009.02911.x

Hultman, J., Vasara, T., Partanen, P. Kurola, J., Kontro, M. H., Paulin, L., et al. (2010). Determination of fungal succession during municipal solid waste composting using a cloning-based analysis. J. Appl. Microbiol. 108, 472-487. doi: 10.1111/j.1365-2672.2009.04439.x

Kadarmoidheen, M., Saranraj, P., and Stella, D. (2012). Effect of cellulolytic fungi on the degradation of cellulosis agricultural wastes. Int. J. Appl. Microbiol. Sci. 1, 213-223.

Karsch-Mizrachi, I., Nakamura, Y., and Cochrane, G. (2011). The international nucleotide sequence database collaboration. Nucleic Acids Res. 40, D33-D37. doi: 10.1093/nar/gkr1006

Latge, J. (1999). Aspergillus fumigatus and aspergillosis. Clin. Microbiol. Rev. 12, 310-350. 
Lentendu, G., Zinger, L., Manel, S., Coissac, E., Choler, P., Geremia, A., et al. (2011). Assessment of soil fungal diversity in different alpine tundra habitats by means of pyrosequencing. Fungal Divers. 29, 113-123. doi: 10.1007/s13225-0110101-5

McGinnis, M. R., Campbell, G., Gourley, W. K., and Lucia, L. H. (1992). Phaeohyphomycosis caused by Bipolaris spicifera: an informative case. Eur. J. Epidemiol. 8, 383-386. doi: 10.1007/BF00158572

Miyamoto, T., Sasaoka, R., Kawaguchi, M., Ishioka, S., Inoue, T., Yamada, N., et al. (1998). Scedosporium apiospermum skin infection: a case report and review of the literature. J. Amer. Acad. Dermatol. 39, 498-500. doi: 10.1016/S01909622(98)70335-4

Narutaki, S., Takatori, K., Nishimura, H., Terashima, H., and Sasaki, T. (2002). Identification of fungi based on the nucleotide sequence homology of their Internal Transcribed Spacer 1 (ITS1) region. PDA J. Pharm. Sci. Technol. 56, 90-98.

Nilsson, R. H., Bok, G., Ryberg, M., Kristiansson, E., and Hallenberg, N. (2009). A software pipeline for processing and identification of fungal ITS sequences. Source Code Biol. Med. 4:1. doi: 10.1186/1751-0473-4-1

Nilsson, R. H., Kristiansson, E., Ryberg, M., Hallenberg, N., and Larsson, K.H. (2008). Intraspecific ITS variability in the kingdom fungi as expressed in the international sequence databases and its implications for molecular species identification. Evol. Bioinform. 4, 193-201.

Odds, F. C., Van Gerven, F., EspinelIngroff, A., Bartlett, M. S., Ghannoum, M. A., Lancaster, M. V., et al. (1998). Evaluation of possible correlations between antifungal susceptibilities of filamentous fungi in vitro and antifungal treatment outcomes in animal infection models. Antimicrob. Agents Chemother. 42, 282.

Ogden, P. E., Hurley, D. L., and Cain, P. T. (1992). Fatal fungal endarteritis caused by Bipolaris spicifera following replacement of the aortic valve. Clin. Infect. Dis. 14, 596-598. doi: 10.1093/clinids/14.2.596

Orgiazzi, A., Lumini, E., Nilsson, R. H., Girlanda, M., Vizzini, A., Bonfante, P., et al. (2012). Unravelling soil fungal communities from different Mediterranean land-use backgrounds. PLoS ONE 7:e34847. doi: 10.1371/journal.pone.0034847

Partanen, P., Hultman, J., Paulin, L., Auvinen, P., and Romantschuk, M. (2010). Bacterial diversity at different stages of the composting process. BMC Microbiol. 10:94. doi: 10.1186/1471-2180-10-94

Pipenbring, O. A., Caceres Mendez, A. A., Espino, E., Kirschna, R., and Schofer, H. (2007). Chromoblastomycosis caused by Chaetomium funicola: a case report from western Panama. $\mathrm{Br}$. J. Dermatol. 157, 1025-1029. doi: 10.1111/j.1365-2133.2007.08091.x

Revankar, G. S., Patterson, E. J., Sutton, A. D., Pullen, R., and Rinaldi, G. M. (2002). Disseminated phaeohyphomycosis: review of an emerging mycosis. Clin. Infect. Dis. 34, 467-476. doi: 10.1086/338636

Ryckeboer, J., Mergaert, J., Coosemans, J., Deprins, K., and Swings, J. (2003). Microbiological aspects of biowaste during composting in a monitored compost bin. J. Appl. Microbiol. 94, 127-137. doi: 10.1046/j.1365-2672.2003.01800.x

Sharma, H. S. S. (1989). Economic importance of thermophilus fungi. Appl. Micorbiol. Biotechnol. 31, 1-10. doi: 10.1007/BF00252517

Silva, J. P., Alviano, D. S., Alviano, C. S., De Souza, W., Travassos,
L. R., Diniz, J. A., et al. (2002). Comparison of Fonsecaea pedrosoi sclerotic cells obtained in vivo and in vitro: ultrastructure and antigenicity. FEMS Immunol. Med. Microbiol. 33, 63-69. doi 10.1016/S0928-8244(02)00278-X

Smith, B., and Wilson, J. B. (1996). A consumer's guide to evenness measures. Oikos 76, 70-82. doi: $10.2307 / 3545749$

Takaku, H., Kodaira, S., Kimoto, A. Nashimoto, M., and Takagi, M. (2006). Microbial communities in the garbage composting with rice hull as an amendment revealed by culture-dependent and independent approaches. J. Biosci. Bioeng. 101, 42-50. doi: 10.1263/ jbb.101.42

Tedersoo, L., Nilsson, R. H., Abarenkov, K., Jairus, T., Sadam, A., Saar, I., et al. (2010). 454 Pyrosequencing and Sanger sequencing of tropical mycorrhizal fungi provide similar results but reveal substantial methodological biases. New Phytol. 188, 291-301. doi: 10.1111/j.1469-8137. 2010.03373.x

Thummes, K., Kampfer, P., and Jackel, U. (2007). Temporal change of composition and potential activity of the thermophilic archaeal community during the composting of organic material. Syst. Appl. Microbiol. 30, 418-429. doi: 10.1016/j.syapm.2007.01.006

Tiquia, S. M. (2005). Microbial community dynamics in manure composts based on $16 \mathrm{~S}$ and $18 \mathrm{~S}$ rDNA T-RFLP profiles. Environ. Technol. 26, 1101-1113. doi: 10.1080/09593332608618482

Tokeshi, M. (1993). Species abundance patterns and community structure. Adv. Ecol. Res. 24, 112-186. doi: 10.1016/S0065-2504 (08)60042-2

Wiegant, W. M. (1992). Growth characteristics of the thermophilic fungus
Scytalidium thermophilum in relation to production of mushroom compost. Appl. Environ. Microbiol. 58, 1301-1307.

Wingard, J. R., Merz, W. G., and Saral, R. (1979). Candida tropicalis: a major pathogen in immunocompromised patients. Ann. Intern. Med. 94, 539-543. doi: 10.7326/0003-4819-91-4-539

$\mathrm{Xu}$, L., Ravnskov, S., Larsen, J., Nilsson, R. H., and Nicolaisen, M. (2012). Soil fungal community structure along a soil health gradient in pea fields examined using deep amplicon sequencing. Soil Biol. Biochem. 46, 26-32. doi 10.1016/j.soilbio.2011.11.010

Conflict of Interest Statement: The authors declare that the research was conducted in the absence of any commercial or financial relationships that could be construed as a potential conflict of interest.

Received: 12 March 2013; accepted: 03 June 2013; published online: 13 June 2013.

Citation: De Gannes V, Eudoxie G and Hickey WJ (2013) Insights into fungal communities in composts revealed by 454-pyrosequencing: implications for human health and safety. Front. Microbiol. 4:164. doi: 10.3389/fmicb. 2013.00164

This article was submitted to Frontiers in Microbiotechnology, Ecotoxicology and Bioremediation, a specialty of Frontiers in Microbiology.

Copyright () 2013 De Gannes, Eudoxie and Hickey. This is an open-access article distributed under the terms of the Creative Commons Attribution License, which permits use, distribution and reproduction in other forums, provided the original authors and source are credited and subject to any copyright notices concerning any third-party graphics etc. 